\title{
Water-Insoluble Organics Dominate Brown Carbon in Wintertime Urban Aerosol of China: Chemical Characteristics and Optical Properties
}

Ru-Jin Huang, ${ }^{, \dagger},+, \nabla$ Lu Yang, ${ }^{\dagger}$ Jincan Shen,,${ }^{*} \S$ Wei Yuan, ${ }^{\dagger}$ Yuquan Gong, ${ }^{\dagger}$ Jie Guo, ${ }^{\dagger}$ Wenjuan

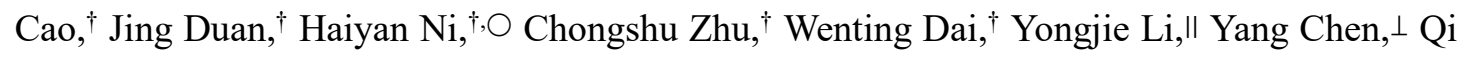
Chen, ${ }^{\#}$ Yunfei Wu, ${ }^{\nabla}$ Renjian Zhang, ${ }^{\nabla}$ Ulrike Dusek, $\bigcirc$ Colin O’Dowd, $\bullet$ Thorsten Hoffmann ${ }^{\nabla}$

${ }^{\dagger}$ State Key Laboratory of Loess and Quaternary Geology, Center for Excellence in Quaternary Science and Global Change, and Key Laboratory of Aerosol Chemistry and Physics, Institute of Earth Environment, Chinese Academy of Sciences, Xi'an 710061, People's Republic of China

'Institute of Global Environmental Change, Xi' an Jiaotong University, Xi' an 710049, China

$\nabla$ Open Studio for Oceanic-Continental Climate and Environment Changes, Pilot National Laboratory for Marine Science and Technology (Qingdao), Qingdao 266061, People's Republic of China

${ }^{\S}$ Key Laboratory of Detection Technology R \& D on Food Safety, Food Inspection and Quarantine Technology Center of Shenzhen Customs, Shenzhen 518045, People's Republic of China

IIDepartment of Civil and Environmental Engineering, Faculty of Science and Technology, University of Macau, Taipa, Macau 999078, People's Republic of China

${ }^{\perp}$ Chongqing Institute of Green and Intelligent Technology, Chinese Academy of Sciences, Chongqing 400714, People's Republic of China

\#State Key Joint Laboratory of Environmental Simulation and Pollution Control, College of Environmental Sciences and Engineering, Peking University, Beijing 100871, People's Republic of China

${ }^{\nabla}$ RCE-TEA, Institute of Atmospheric Physics, Chinese Academy of Sciences, Beijing 100029, People's Republic of China

OCentre for Isotope Research (CIO), Energy and Sustainability Research Institute Groningen (ESRIG), University of Groningen, 9747 A Groningen, The Netherlands

-School of Physics and Centre for Climate and Air Pollution Studies, Ryan Institute, National University of Ireland Galway, University Road, Galway H91CF50, Ireland

VInstitute of Inorganic and Analytical Chemistry, Johannes Gutenberg University of Mainz, Duesbergweg 10-14, Mainz 55128, Germany

Corresponding author: E-mail: rujin.huang@ieecas.cn (R.-J.H) or jincansh@263.net (J.S) 
Ions measurement A portion of quartz-fiber filter $\left(10.8 \mathrm{~cm}^{2}\right)$ was extracted with $10 \mathrm{~mL}$ of distilled deionized water. The extracts were filtered through $0.45-\mathrm{mm}$ pore size microporous membranes to remove insoluble material. Inorganic ions including $\mathrm{NO}_{3}^{-}, \mathrm{SO}_{4}{ }^{2-}, \mathrm{Cl}^{-}, \mathrm{Na}^{+}, \mathrm{K}^{+}, \mathrm{Mg}^{2+}$, $\mathrm{Ca}^{2+}$ and $\mathrm{NH}_{4}{ }^{+}$by high-performance anion exchange chromatography with pulsed amperometric detector (HPAEC-PAD) on a Dionex DX-600 ion chromatograph (Dionex Inc., Sunnyvale, CA, USA). IonPac CS12A and AS14A columns were used for the separation of cations and anions, respectively. For anion separation, an eluent of $8 \mathrm{mM} \mathrm{Na}_{2} \mathrm{CO}_{3}$ and $1 \mathrm{mM} \mathrm{NaHCO}_{3}$ was used with a flow rate of $1 \mathrm{~mL} \mathrm{~min}{ }^{-1}$, whereas $20 \mathrm{mM}$ methanesulfonic acid with a flow rate of $1 \mathrm{~mL} \mathrm{~min}^{-1}$ was utilized as eluent for cation separation. Data reported are the field blank corrected. Quality assurance/quality control (QA/QC) procedures are as described by Zhang et al. (2011).

Zhang, T.; Cao, J. J.; et al. Water-soluble ions in atmospheric aerosols measured in Xi'an, China: Seasonal variations and sources. Atmos. Res. 2011, 102,110-119. 
Table S1. HPLC retention times of the nitro-phenols and PAHs standard compounds.

\begin{tabular}{lclc}
\hline Standard compounds & RT (min) & Standard compounds & RT (min) \\
\hline Nitro-phenols & & PAHs & \\
4-nitrocatechol & 7.77 & Naphthalene & 41.59 \\
3-nitrosalicylicacid & 8.63 & Acenaphthylene & 44.52 \\
4-nitrophenol & 12.42 & Acenaphthene & 48.05 \\
5-nitrosalicylicacid & 13.74 & Phenanthrene & 49.70 \\
4-methyl-5-nitrocatechol & 14.94 & Anthracene & 50.52 \\
3,4-dinitrophenol & 19.85 & Fluoranthene & 52.38 \\
3-methyl-5-nitrocatechol & 20.50 & Pyrene & 53.00 \\
3-methyl-4-nitrophenol & 22.06 & Chrysene & 55.42 \\
4-methyl-3-nitrophenol & 24.18 & Benzo(a)anthracene & 55.69 \\
3-methyl-6-nitrocatechol & 24.60 & Benzo(j)fluoranthene & 57.42 \\
2-methyl-4-nitrophenol & 25.64 & Benzo(b)fluoranthene & 57.89 \\
2,6-dimethyl-4-nitrophenol & 31.69 & Benzo(k)fluoranthene & 58.11 \\
4-nitro-1-naphthol & 37.52 & Benzo(a)pyrene & 58.48 \\
& & Dibenzo(a,h)anthracene & 59.79 \\
& & Indeno(1,2,3-cd)pyrene & 60.68 \\
\hline
\end{tabular}




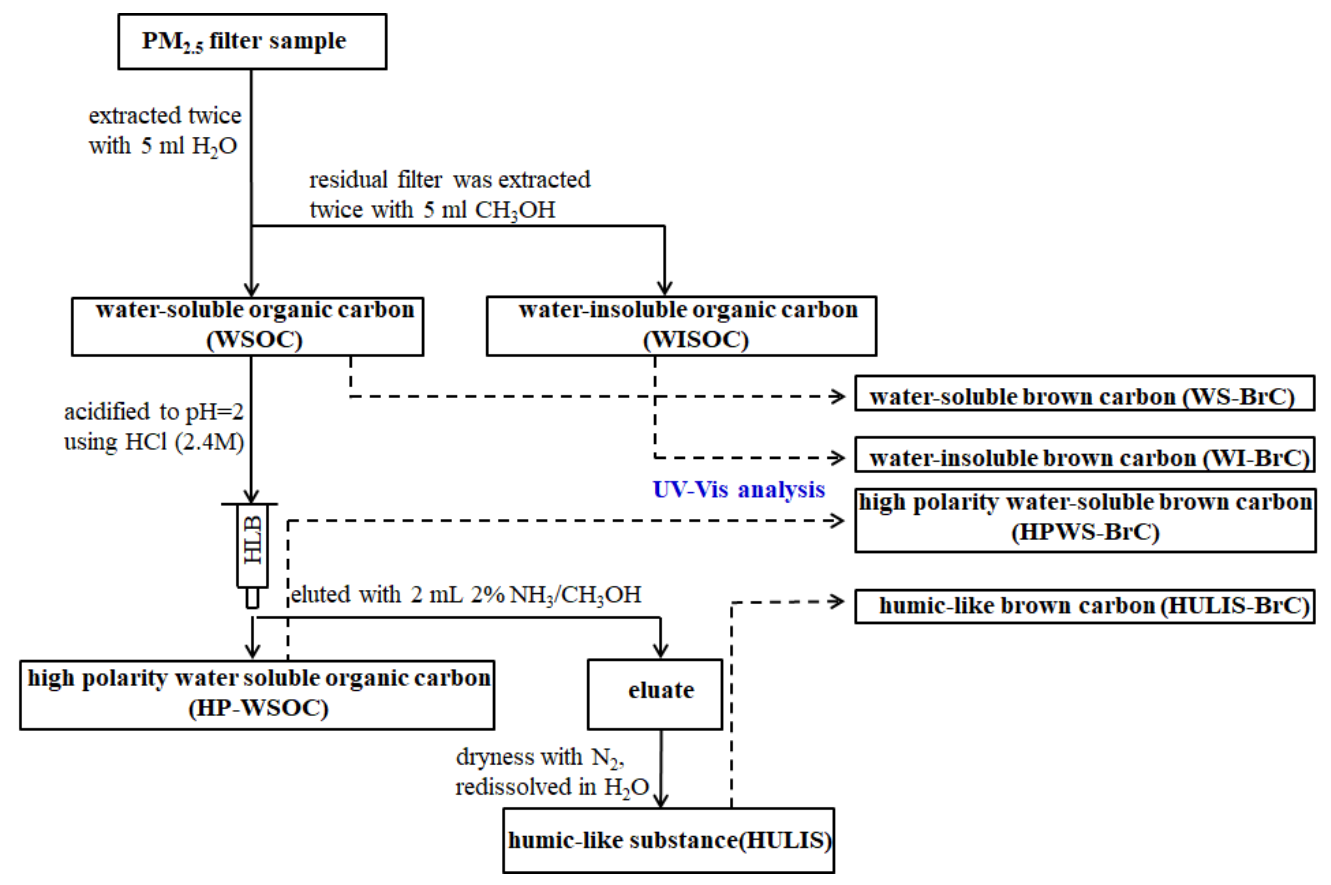

Fig. S1. The procedure of sample extraction and the corresponding abbreviations of $\mathrm{OC}$ and $\mathrm{BrC}$ fractions

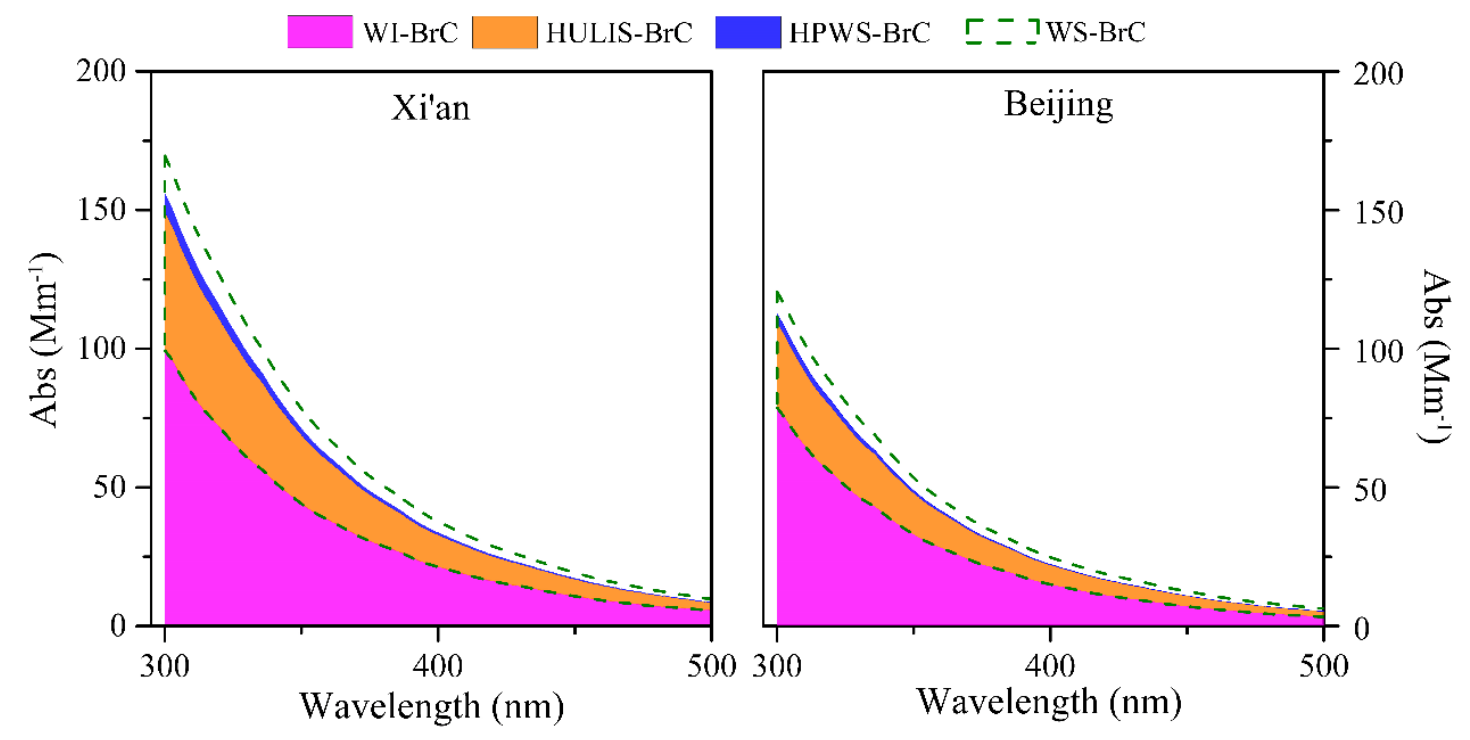

Fig. S2. Light absorption spectra of different $\mathrm{BrC}$ fractions in Xi'an and Beijing. 


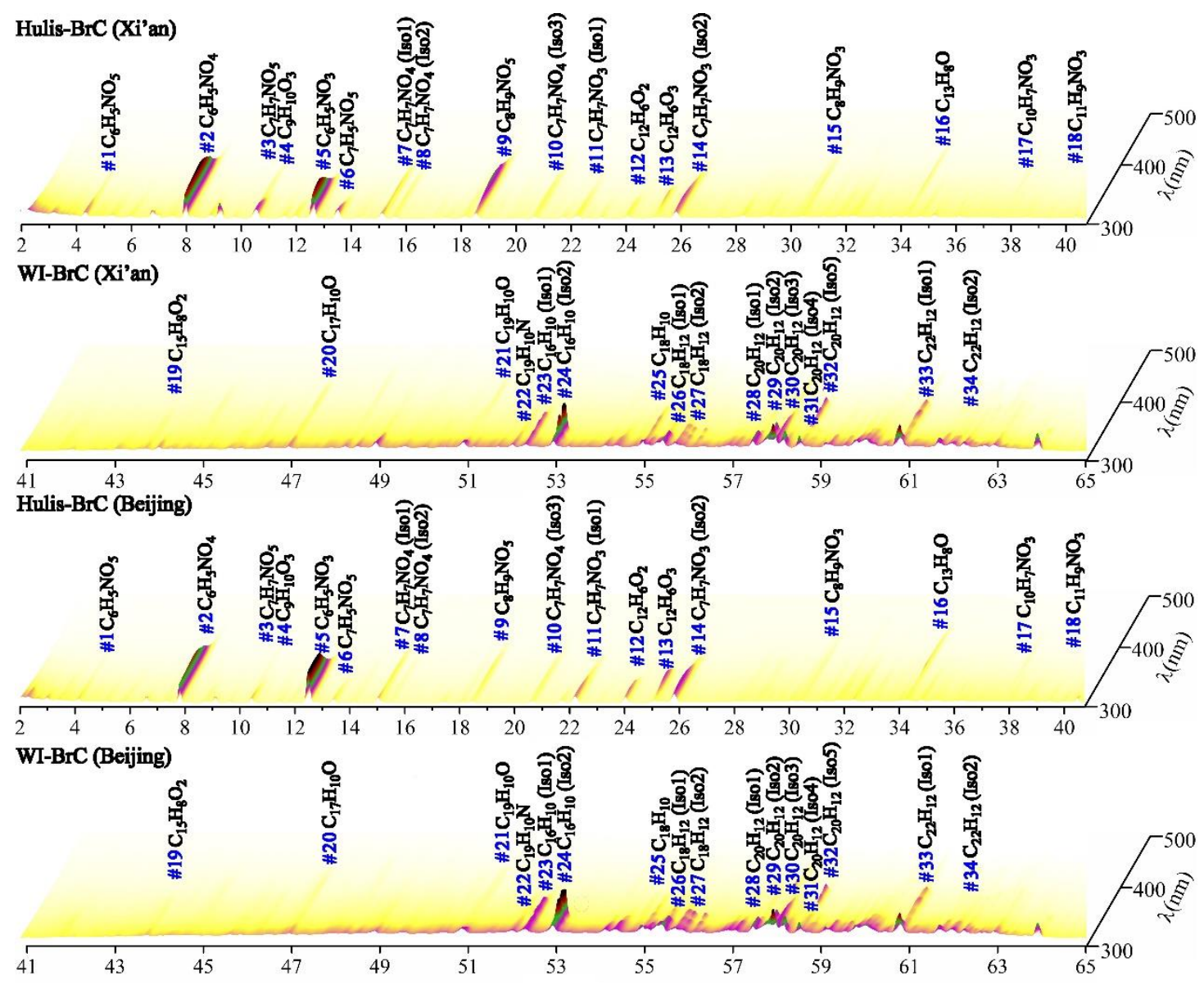

Fig. S3. 3D-plot of HPLC/PDA chromatograms of BrC fractions in Xi'an and Beijing. The color denotes the relative absorbance intensity recorded by the PDA detector. The chemical formulas of the identified main $\mathrm{BrC}$ chromophores are shown above the peaks. 